\title{
FINITE FUNCTORIAL SEMI-NORMS AND REPRESENTABILITY
}

\author{
CLARA LÖH
}

\begin{abstract}
Functorial semi-norms are semi-normed refinements of functors such as singular (co)homology. We investigate how different types of representability affect the (non-)triviality of finite functorial semi-norms on certain functors or classes. In particular, we consider representable functors, generalised cohomology theories, and so-called weakly flexible homology classes in singular homology and $\ell^{1}$-homology.
\end{abstract}

\section{INTRODUCTION}

Functorial semi-norms are semi-normed refinements of functors to the category of Abelian groups (Definition 2.2). Gromov introduced the notion of functorial semi-norms on singular (co)homology [13, 12] in the context of simplicial volume and its relation with geometry and rigidity. For example, the $\ell^{1}$-semi-norm on singular homology is a finite functorial semi-norm on singular homology (Example 2.5). Dually, the $\ell^{\infty}$-semi-norm on singular cohomology (Example 2.9) is a functorial semi-norm on singular cohomology, which is not finite; in contrast, by construction, the $\ell^{\infty}$-semi-norm on bounded cohomology (Example 2.11) is a finite functorial semi-norm on bounded cohomology. Applications of functorial semi-norms in manifold topology include degree theorems [12, 16, 21]. Conversely, knowledge about mapping degrees allows to construct functorial semi-norms with interesting properties [9, 15].

We investigate the relation between (non-)triviality of finite functorial semi-norms and so-called weak flexibility:

Definition 1.1 (weakly flexible). Let $C$ be a category, let $F: C \longrightarrow A b$ be a functor, and let $X \in \mathrm{Ob}(C)$. An element $\alpha \in F(X)$ is weakly flexible (with respect to $F)$ if there exist $Y \in \mathrm{Ob}(C)$ and $\beta \in F(Y)$ such that the set

$$
D(\beta, \alpha):=\left\{d \in \mathbb{Z} \mid \exists_{f \in \operatorname{Mor}_{\mathcal{C}}(Y, X)} F(f)(\beta)=d \cdot \alpha\right\}
$$

is infinite.

It is a simple, yet fundamental, observation that any finite homogeneous functorial semi-norm is trivial on weakly flexible classes (Proposition 3.4).

In this article, we give equivalent descriptions of weak flexibility in singular homology (Section 5.1) and $\ell^{1}$-homology (Section 5.2). Moreover, we apply the above observation to representable functors (Section 4.1) and countably additive functors (Section 4.2). This shows that many classical functors from algebraic topology do not admit any non-trivial finite homogeneous functorial semi-norms. For instance, this might be interesting for the study of comparison maps in bounded cohomology.

Date: March 23, 2022. (C) C. Löh 2014. This work was supported by the CRC 1085 Higher Invariants (Universität Regensburg).

MSC 2010 classification: 55N10, 55N35, 57N65. 


\section{FUNCTORIAL SEMI-NORMS}

In this section, we introduce some basic notation and give a definition of functorial semi-norms, generalising Gromov's concept of functorial seminorms on singular (co)homology:

Definition 2.1 (semi-norms on Abelian groups).

- A semi-norm on an Abelian group $A$ is a map $|\cdot|: A \longrightarrow \mathbb{R}_{\geq 0} \cup\{\infty\}$ with the following properties:

- We have $|0|=0$.

- For all $x \in A$ we have $|-x|=|x|$.

- For all $x, y \in A$ we have $|x+y| \leq|x|+|y|$. Here, $x+\infty:=\infty$ for all $x \in \mathbb{R}_{\geq 0} \cup\{\infty\}$.

Such a semi-norm is finite if the value $\infty$ is not in the image. The semi-norm is homogeneous if for all $n \in \mathbb{Z} \backslash\{0\}$ and all $x \in A$ we have $|n \cdot x|=|n| \cdot|x|$, where $|n|$ denotes the ordinary absolute value on $\mathbb{Z}$. Here, $n \cdot \infty:=\infty$ for all $n \in \mathbb{N} \backslash\{0\}$.

- A (finitely) semi-normed Abelian group is an Abelian group together with a (finite) semi-norm.

- We write snAb for the category of semi-normed Abelian groups, where the morphisms are group homomorphisms that are normnon-increasing.

- We write $s n A b^{\text {fin }}$ for the category of finitely semi-normed Abelian groups, where the morphisms are group homomorphisms that are norm-non-increasing.

Definition 2.2 (functorial semi-norm). Let $C$ be a category, let $F: C \longrightarrow A b$ be a functor (possibly contravariant).

- A functorial semi-norm on $F$ is a factorisation functor $\widehat{F}: C \longrightarrow \operatorname{snAb}$ of $F$ through the forgetful functor snAb $\longrightarrow A b$ :

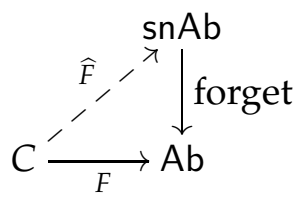

- A finite functorial semi-norm on $F$ is a factorisation $\widehat{F}: C \longrightarrow s n A b^{\text {fin }}$ of $F$ through the forgetful functor snAb fin $\longrightarrow A b$ :

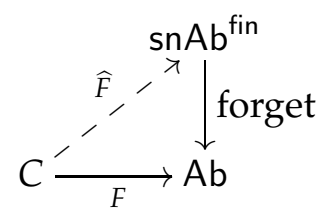

More explicitly, a [finite] functorial semi-norm on a functor $F: C \longrightarrow A b$ consists of a choice of a [finite] semi-norm $|\cdot|$ on $F(X)$ for every $X \in \mathrm{Ob}(C)$ such that for all morphisms $f: X \longrightarrow Y$ in $C$ and all $\alpha \in F(X)$ we have

$$
|F(f)(\alpha)| \leq|\alpha| \text {. }
$$


A functorial semi-norm on $F$ is homogeneous if for all $X \in \mathrm{Ob}(C)$ the corresponding semi-norm on $F(X)$ is homogeneous. Notice that all homogeneous (functorial) semi-norms are trivial on torsion elements.

Example 2.3 (trivial functorial semi-norm). If $C$ is a category and $F: C \longrightarrow$ $\mathrm{Ab}$ is a functor, then equipping for every $X \in \mathrm{Ob}(C)$ the group $F(X)$ with the zero semi-norm gives a functorial semi-norm on $F$, the trivial functorial semi-norm on F.

2.1. $\ell^{1}$-Homology. A key example is given by the $\ell^{1}$-semi-norm on homology of simplicial sets; the $\ell^{1}$-semi-norm on singular homology and the $\ell^{1}$ semi-norm on group homology are instances of this construction:

Example 2.4 ( $\ell^{1}$-semi-norm on $\left(\ell^{1}\right)$-homology of simplicial sets). Let $\Delta$ be the simplex category: The objects of $\Delta$ are natural numbers; for $n, m \in \mathbb{N}$ the set of morphisms $n \longrightarrow m$ in $\Delta$ is the set of monotone (increasing) functions of type $\{0, \ldots, n\} \longrightarrow\{0, \ldots, m\}$, and composition of morphisms in $\Delta$ is given by the set-theoretic composition of the underlying functions. The category of simplicial sets is denoted by $\Delta$ (Set); objects are contravariant functors $\Delta \longrightarrow$ Set and morphisms are natural transformations of such functors. If $R$ is a ring (with unit), then there is the corresponding chain complex functor

$$
\mathrm{Ch}_{R}: \Delta(\text { Set }) \longrightarrow \mathrm{Ch}_{R} \longrightarrow \mathrm{Ch}_{\mathbb{Z}}
$$

into the category of $R$-chain complexes [11] followed by the forgetful functor into $\mathbb{Z}$-chain comlexes. By construction, for a simplicial set $S$ and $n \in \mathbb{N}$, the chain module $\mathrm{Ch}_{R}(S)_{n}$ is the free $R$-module with basis $S(n)$. If $R$ (as Abelian group) is equipped with a norm, then $\mathrm{Ch}_{R}(S)_{n}$ inherits a finite norm $|\cdot|_{1, R}$ : the $\ell^{1}$-norm with respect to the basis $S(n)$. This norm in turn leads to the $\ell^{1}$-semi-norm on homology in degree $n$ :

$$
\begin{aligned}
\|\cdot\|_{1, R}: H_{n}\left(\mathrm{Ch}_{R}(S)\right) & \longrightarrow \mathbb{R}_{\geq 0} \\
\alpha & \longmapsto \inf \left\{|c|_{1, R} \mid c \in \mathrm{Ch}_{R}(S)_{n}, \partial c=0,[c]=\alpha\right\} .
\end{aligned}
$$

By definition of $|\cdot|_{1, R}$, the functor $\mathrm{Ch}_{R}$ turns natural transformations into norm-non-increasing chain maps. Applying homology in degree $n$ hence yields a finite functorial semi-norm on the composition

$$
H_{n} \circ \mathrm{Ch}_{R}: \Delta(\text { Set }) \longrightarrow \mathrm{Ab} .
$$

By construction of the chain complex functor $\mathrm{Ch}_{R}$, the boundary operators in the chain complex $\mathrm{Ch}_{R}(S)$ are bounded with respect to the $\ell^{1}$-norm. Hence, completing with respect to $|\cdot|_{1, R}$ (and extending the boundary operators continuously) leads to a functor

$$
\mathrm{Ch}_{R}^{\ell^{1}}: \Delta(\text { Set }) \longrightarrow \mathrm{Ch}_{\mathbb{Z}} .
$$

Clearly, the $\ell^{1}$-norm on the chain modules of $\mathrm{Ch}_{R}(S)$ extends to an $\ell^{1}$-norm on the chain modules of $\mathrm{Ch}_{R}^{\ell^{1}}(S)$, which we will also denote by $\left.|\cdot|\right|_{1, R}$. Similarly to the uncompleted case, we then obtain a finite functorial $\ell^{1}$-seminorm on the $\ell^{1}$-homology functor

$$
H_{n}^{\ell^{1}} \circ \mathrm{Ch}_{R}=H_{n} \circ \mathrm{Ch}_{R}^{\ell^{1}}: \Delta(\text { Set }) \longrightarrow \mathrm{Ab}
$$


in degree $n$.

In all these cases, we abbreviate $\|\cdot\|_{1}:=\|\cdot\|_{1, \mathbb{R}}$. Notice that if $\mathbb{Q}$ is a subring of $R$, then these functorial semi-norms on $\left(\ell^{1}-\right)$ homology are homogeneous. In general, $\|\cdot\|_{1 . Z}$ is not homogeneous.

Example 2.5 ( $\ell^{1}$-semi-norm on singular homology). Let $R$ be a normed ring, and let $n \in \mathbb{N}$. Then singular homology $H_{n}(\cdot ; R)$ in degree $n$ of topological spaces with coefficients in $R$ can be described as the composition

$$
H_{n} \circ \mathrm{Ch}_{R} \circ S: \text { Top } \longrightarrow \Delta(\text { Set }) \longrightarrow \mathrm{Ch}_{\mathbb{Z}} \longrightarrow \mathrm{Ab},
$$

where $S:$ Top $\longrightarrow \Delta$ (Set) denotes the total singular complex functor [11]. So, $H_{n}(\cdot ; R)$ inherits a finite functorial semi-norm, the $\ell^{1}$-semi-norm $\|\cdot\|_{1, R}$. More explicitly, $\|\cdot\|_{1, R}$ is given as follows: If $X$ is a topological space and $\alpha \in$ $H_{n}(X ; R)$, then

$$
\|\alpha\|_{1, R}=\inf \left\{\sum_{j=0}^{k}\left|a_{j}\right| \mid \sum_{j=0}^{k} a_{j} \cdot \sigma_{j} \in C_{n}(X ; R) \text { is a cycle representing } \alpha\right\},
$$

where $|\cdot|$ denotes the norm on $R$.

An example of a topological invariant defined in terms of the $\ell^{1}$-seminorm on singular homology with $\mathbb{R}$-coefficients is the simplicial volume [12. 17]: The simplicial volume of an oriented closed connected $n$-manifold $M$ is

$$
\|M\|:=\left\|[M]_{\mathbb{R}}\right\|_{1} \in \mathbb{R}_{\geq 0},
$$

where $[M]_{\mathbb{R}} \in H_{n}(M ; \mathbb{R})$ is the $\mathbb{R}$-fundamental class of $M$. For example, the simplicial volume of hyperbolic manifolds is non-zero [12, 26, 5]. Classical applications of simplicial volume include Gromov's proof of Mostow rigidity [23] and degree theorems in the presence of sufficiently negative curvature [12, 16, 21].

Functorial semi-norms on singular homology that differ essentially from the $\ell^{1}$-semi-norm can be constructed via manifold topology [9, 15]. However, it remains an open problem to determine whether there are also finite functorial semi-norms on singular homology that are not dominated in some weak sense (e.g., through a monotonic function) by the $\ell^{1}$-semi-norm.

Example 2.6 ( $\ell^{1}$-semi-norm on group homology). Let $R$ be a normed ring, and let $n \in \mathbb{N}$. Similarly to the previous example, homology of groups in degree $n$ with coefficients in $R$ can be described as the composition

$$
H_{n} \circ \mathrm{Ch}_{R} \circ B: \text { Group } \longrightarrow \Delta(\text { Set }) \longrightarrow \mathrm{Ch}_{\mathbb{Z}} \longrightarrow \mathrm{Ab},
$$

where $B$ : Group $\longrightarrow \Delta$ (Set) denotes the simplicial classifying space functor [11]. Hence, also group homology in degree $n$ with coefficients in $R$ inherits a functorial $\ell^{1}$-semi-norm $\|\cdot\|_{1, R}$.

The canonical isomorphism between group homology via the simplicial classifying space functor and singular homology of Eilenberg-MacLane spaces of type $K(\cdot, 1)$ is isometric with respect to $\|\cdot\|_{1, R}$, as can be seen by constructing explicit mutually inverse chain homotopy equivalences. 
Example 2.7 ( $\ell^{1}$-semi-norm on $\ell^{1}$-homology). Let $R$ be a normed ring, and let $n \in \mathbb{N}$. Then $\ell^{1}$-homology of spaces or groups respectively in degree $n$ with coefficients in $R$ are defined by

$$
\begin{aligned}
& H_{n}^{\ell^{1}}(\cdot ; R):=H_{n}^{\ell^{1}} \circ \mathrm{Ch}_{R} \circ S: \text { Top } \longrightarrow \Delta(\text { Set }) \longrightarrow \mathrm{Ab}, \\
& H_{n}^{\ell^{1}}(\cdot ; R):=H_{n}^{\ell^{1}} \circ \mathrm{Ch}_{R} \circ B: \text { Group } \longrightarrow \Delta(\text { Set }) \longrightarrow \mathrm{Ab} .
\end{aligned}
$$

As in Example 2.4, these functors admit functorial $\ell^{1}$-semi-norms $\|\cdot\|_{1, R}$. For more information about $\ell^{1}$-homology and its applications we refer to the literature [18, 6, 4, 20].

2.2. Bounded cohomology. Dually, we can also equip cohomology of simplicial sets with a functorial semi-norm - the $\ell^{\infty}$-semi-norm, which in turn leads to bounded cohomology:

Example 2.8 ( $\ell^{\infty}$-semi-norm on (bounded) cohomology of simplicial sets). Let $R$ be a normed ring. Then there is the dual cochain complex functor

$$
\mathrm{Ch}_{R}^{*}:=\operatorname{Hom}_{\mathbb{Z}}(\cdot, R) \circ \mathrm{Ch}_{\mathbb{Z}}: \Delta(\mathrm{Set}) \longrightarrow \mathrm{CoCh}_{R} \longrightarrow \mathrm{CoCh}_{\mathbb{Z}}
$$

as well as the topological dual cochain complex functor

$$
\mathrm{Ch}_{R}^{\#}:=B(\cdot, R) \circ \mathrm{Ch}_{\mathbb{Z}}: \Delta(\text { Set }) \longrightarrow \mathrm{CoCh}_{R} \longrightarrow \mathrm{CoCh}_{\mathbb{Z}}
$$

of bounded $\mathbb{Z}$-linear functionals; both these functors are contravariant. If $S$ is a simplicial set and $n \in \mathbb{N}$, then the modules $\operatorname{Ch}_{R}^{*}(S)^{n}$ and $\operatorname{Ch}_{R}^{\#}(S)^{n}$ inherit a norm $|\cdot|_{\infty, R}$ : the $\ell^{\infty}$-norm on linear functionals with respect to the $\ell^{1}$-norm on $\mathrm{Ch}_{\mathbb{Z}}(S)_{n}$; this norm on $\mathrm{Ch}_{R}^{*}(S)^{n}$, in general, is not finite, but by construction - this norm on $\mathrm{Ch}_{R}^{\#}(S)^{n}$ is finite. These norms in turn lead to the $\ell^{\infty}$-semi-norm on cohomology in degree $n$

$$
\begin{aligned}
\|\cdot\|_{\infty, R}: H^{n}\left(\mathrm{Ch}_{R}^{*}(S)\right) & \longrightarrow \mathbb{R}_{\geq 0} \cup\{\infty\} \\
\varphi & \longmapsto \inf \left\{|f|_{\infty, R} \mid f \in \mathrm{Ch}_{R}^{*}(S)^{n}, \delta f=0,[f]=\varphi\right\},
\end{aligned}
$$

and the $\ell^{\infty}$-semi-norm on bounded cohomology in degree $n$ :

$$
\begin{aligned}
\|\cdot\|_{\infty, R}: H^{n}\left(\mathrm{Ch}_{R}^{\#}(S)\right) & \longrightarrow \mathbb{R}_{\geq 0} \\
\varphi & \longmapsto \inf \left\{|f|_{\infty, R} \mid f \in \mathrm{Ch}_{R}^{\#}(S)^{n}, \delta f=0,[f]=\varphi\right\} .
\end{aligned}
$$

By definition of $|\cdot|_{\infty, R}$, the functors $\mathrm{Ch}_{R}^{*}$ and $\mathrm{Ch}_{R}^{\#}$ turn natural transformations into norm-non-increasing chain maps. Applying (co)homology in degree $n$ hence yields a finite functorial semi-norm on the contravariant compositions

$$
\begin{aligned}
& H^{n} \circ \mathrm{Ch}_{R}^{*}: \Delta(\text { Set }) \longrightarrow \mathrm{Ab}, \\
& H^{n} \circ \mathrm{Ch}_{R}^{\#}: \Delta(\text { Set }) \longrightarrow \mathrm{Ab} .
\end{aligned}
$$

In all theses cases, we abbreviate $\|\cdot\|_{\infty}:=\|\cdot\|_{\infty, \mathbb{R}}$. If $\mathbb{Q}$ is a subring of $R$, then these functorial semi-norms on (bounded) cohomology are homogeneous. In general, $\|\cdot\|_{\infty, \mathbb{Z}}$ is not homogeneous.

Example 2.9 ( $\ell^{\infty}$-semi-norm on singular cohomology). Let $R$ be a normed ring, and let $n \in \mathbb{N}$. Then singular cohomology $H^{n}(\cdot ; R)$ in degree $n$ of 
topological spaces with coefficients in $R$ can be described as the composition

$$
H^{n} \circ \mathrm{Ch}_{R}^{*} \circ S: \text { Top } \longrightarrow \Delta(\text { Set }) \longrightarrow \mathrm{CoCh}_{\mathbb{Z}} \longrightarrow \mathrm{Ab},
$$

where $S:$ Top $\longrightarrow \Delta$ (Set) denotes the total singular complex functor. So, $H^{n}(\cdot ; R)$ inherits a functorial semi-norm, the $\ell^{\infty}$-semi-norm $\|\cdot\|_{\infty, R}$. More explicitly, $\|\cdot\|_{\infty, R}$ is given as follows: If $X$ is a topological space and $\varphi \in$ $H^{n}(X ; R)$, then

$\|\varphi\|_{\infty, R}=\inf \left\{\sup _{\sigma \in \operatorname{map}\left(\Delta^{n}, X\right)}|f(\sigma)| \mid f \in C^{n}(X ; R)\right.$ is a cocycle representing $\left.\varphi\right\}$,

where $|\cdot|$ denotes the norm on $R$.

Example 2.10 ( $\ell^{\infty}$-semi-norm on group cohomology). Let $R$ be a normed ring, and let $n \in \mathbb{N}$. As in the previous examples, cohomology of groups in degree $n$ with coefficients in $R$ inherits a functorial $\ell^{\infty}$-semi-norm $\|\cdot\|_{\infty, R}$, based on the simplicial classifying space functor $B$ : Group $\longrightarrow \Delta$ (Set).

Example 2.11 ( $\ell^{\infty}$-semi-norm on bounded cohomology). Let $R$ be a normed ring, and let $n \in \mathbb{N}$. Then bounded cohomology of spaces or groups respectively in degree $n$ with coefficients in $R$ are defined by

$$
\begin{aligned}
& H_{b}^{n}(\cdot ; R):=H^{n} \circ \mathrm{Ch}_{R}^{\#} \circ S: \text { Top } \longrightarrow \Delta(\text { Set }) \longrightarrow \mathrm{Ab} \\
& H_{b}^{n}(\cdot ; R):=H^{n} \circ \mathrm{Ch}_{R}^{\#} \circ B: \text { Group } \longrightarrow \Delta(\text { Set }) \longrightarrow \mathrm{Ab} .
\end{aligned}
$$

As described in Example 2.8, these functors admit finite functorial $\ell^{\infty}$-seminorms $\|\cdot\|_{\infty, R}$.

The inclusion of bounded linear functionals into all linear functionals induces natural transformations $H_{b}^{n}(\cdot ; R) \Longrightarrow H^{n}(\cdot ; R)$ between bounded cohomology of spaces/groups and ordinary cohomology of spaces/groups, the so-called comparison maps. In general, these comparison maps are neither injective nor surjective (Example4.8).

There is a duality principle that allows to express the $\ell^{1}$-semi-norm on homology in terms of the $\ell^{\infty}$-semi-norm of the corresponding bounded cohomology [12]. Therefore, bounded cohomology is an important algebraic tool in the study of simplicial volume. For more information about bounded cohomology and its applications we refer to the literature [12, 14, 22, 6].

\section{WEAK FLEXIBILITY}

We introduce the following notions of representability of classes, generalising (strong) inflexibility of manifolds [9]:

Definition 3.1 ((weakly) flexible). Let $C$ be a category, let $F: C \longrightarrow$ Ab be a functor, let $X \in \mathrm{Ob}(C)$, and let $\alpha \in F(X)$.

- The element $\alpha$ is flexible (with respect to $F$ ) if the set

$$
D(\alpha):=\left\{d \in \mathbb{Z} \mid \exists_{f \in \operatorname{Mor}_{C}(X, X)} F(f)(\alpha)=d \cdot \alpha\right\}
$$

is infinite. Otherwise $\alpha$ is called inflexible. 
- The element $\alpha$ is weakly flexible (with respect to $F$ ) if there exists an object $Y \in \mathrm{Ob}(C)$ and $\beta \in F(Y)$ such that the set

$$
D(\beta, \alpha):=\left\{d \in \mathbb{Z} \mid \exists_{f \in \operatorname{Mor}_{C}(Y, X)} \quad F(f)(\beta)=d \cdot \alpha\right\}
$$

is infinite. Otherwise $\alpha$ is called strongly inflexible.

Example 3.2. For example, fundamental classes of spheres, tori, and (oriented) projective spaces are flexible with respect to singular homology with $\mathbb{Z}$ - or $\mathbb{R}$-coefficients. Not all (fundamental classes of) oriented closed simply connected manifolds are flexible [3, 9, 2, 8]; however, it remains an open problem whether there exist oriented closed simply connected manifolds that are strongly inflexible.

Example 3.3 (torsion classes). Let $C$ be a category and let $F: C \longrightarrow A b$ be a functor. If $X \in \mathrm{Ob}(C)$ and $\alpha \in F(X)$ is a torsion element of order $m$, then $m \cdot \mathbb{Z}+1 \subset D(\alpha)$ and so $\alpha$ is flexible.

In the following, we will make use of the following simple, yet fundamental, observation:

Proposition 3.4 (weak flexibility and finite functorial semi-norms). Let $C$ be a category, let $F: C \longrightarrow \mathrm{Ab}$ be a functor, let $X \in \mathrm{Ob}(C)$ and let $\alpha \in F(X)$ be weakly flexible. If $\widehat{F}: C \longrightarrow \mathrm{snAb}^{\text {fin }}$ is a finite homogeneous functorial semi-norm on $F$, then $|\alpha|_{\widehat{F}}=0$.

Proof. Because $\alpha$ is weakly flexible, there is an object $Y \in \mathrm{Ob}(C)$ and $\beta \in$ $F(Y)$ such that $D(\beta, \alpha)$ is infinite. If $\widehat{F}: C \longrightarrow \operatorname{snAb} b^{\text {fin }}$ is a finite homogeneous functorial semi-norm on $F$, we obtain

$$
|\alpha|_{\widehat{F}} \leq \inf \left\{\frac{1}{d} \cdot|\beta|_{\widehat{F}} \mid d \in D(\beta, \alpha) \backslash\{0\}\right\}=0 .
$$

Example 3.5. In particular, the fundamental class of an oriented closed connected manifold with non-zero simplicial volume ist not weakly flexible with respect to real singular homology. Prominent examples of this type are hyperbolic manifolds [12, 26, 5].

\section{REPRESENTABLE FUNCTORS AND COHOMOLOGY THEORIES}

We will now apply the observation Proposition 3.4 to representable functors (Section 4.1) as well as countably additive functors (Section 4.2).

4.1. Weak flexibility and representable functors. Representable functors do not admit non-trivial finite functorial semi-norms:

Corollary 4.1 (finite functorial semi-norms on representable functors). Let $\mathrm{C}$ be a category and let $\mathrm{F}: \mathrm{C} \longrightarrow \mathrm{Ab}$ be a (co- or contravariant) representable functor. If $\widehat{F}: C \longrightarrow s n A b^{\text {fin }}$ is a finite homogeneous functorial semi-norm on $C$, then $\widehat{F}$ is trivial.

Proof. We give the proof in the covariant case (the contravariant case being dual). By Proposition 3.4, it suffices to establish that all classes are weakly flexible with respect to $F$. Let $Y \in \mathrm{Ob}(C)$ be a representing object of $F$, i.e., $V \circ F \cong \operatorname{Mor}_{C}(Y, \cdot)$, where $V: A b \longrightarrow$ Set is the forgetful functor; let $\beta \in F(Y)$ be the element corresponding to $\operatorname{id}_{Y} \in \operatorname{Mor}_{C}(Y, Y)$. 
Let $X \in \mathrm{Ob}(C)$, let $\alpha \in F(X)$, and let $d \in \mathbb{Z}$. Then $d \cdot \alpha$ corresponds to a morphism $f \in \operatorname{Mor}_{C}(Y, X)$, and so $d \cdot \alpha=F(f)(\beta)$. Thus, $D(\beta, \alpha)=\mathbb{Z}$, which shows that $\alpha$ is weakly flexible with respect to $F$.

Example 4.2 (homotopy groups). Let $n \in \mathbb{N}_{>1}$. Then the $n$-th homotopy group functor

$$
\pi_{n}: \mathrm{Top}_{* \mathrm{~h}} \longrightarrow \mathrm{Ab}
$$

on the homotopy category $\mathrm{Top}_{* \mathrm{~h}}$ of pointed topological spaces is represented by the marked $n$-sphere. Hence, by Corollary 4.1 , the functor $\pi_{n}$ does not admit a non-trivial finite homogeneous functorial semi-norm.

Example 4.3 (cohomology theories on CW-complexes). Cellular cohomology with coefficients in an Abelian group $A$ does not admit a non-trivial finite homogeneous functorial semi-norm in degree $n \in \mathbb{N}$ : The functor $H^{n}(\cdot ; A)$ on the homotopy category $\mathrm{CW}_{\mathrm{h}}$ of $\mathrm{CW}$-complexes is representable by $\mathrm{CW}$-complexes of type $K(A, n)$.

In view of the Brown representability theorem, the same holds for finitely additive generalised cohomology theories on the homotopy category of (pointed) finite $\mathrm{CW}$-complexes that yield finitely generated Abelian groups on spheres [25, Chapter 21.8][1]: In general, the representing object will not necessarily have the homotopy type of a finite CW-complex, but only of a $\mathrm{CW}$-complex $Y$ of finite type. If $X$ is a finite $\mathrm{CW}$-complex of dimension $n$, then the inclusion $Y_{n+1} \hookrightarrow Y$ of the $(n+1)$-skeleton induces a natural bijection $\left[X, Y_{n+1}\right] \longrightarrow[X, Y]$ and we can apply Corollary 4.1 to the restriction of our given cohomology functor $F$ to the full subcategory of $\mathrm{CW}_{\mathrm{h}}$ generated by $X$ and $Y_{n+1}$; on this subcategory, $F$ is naturally isomorphic to $\left[\cdot, Y_{n+1}\right]$ This shows that all classes in $F(X)$ are weakly flexible. Hence, any finite homogeneous functorial semi-norm on $F$ is trivial on $F(X)$.

For example, this applies to complex/real topological K-theory or oriented cobordism.

The case of countably additive cohomology theories can be handled as in Section 4.2 .

Example 4.4 (bounded cohomology of spaces and groups). Let $n \in \mathbb{N}_{>1}$. Then there exist finite $\mathrm{CW}$-complexes $X$ and (finitely presented) groups $G$ respectively such that $\|\cdot\|_{\infty}$ is non-trivial on $H_{b}^{n}(X ; \mathbb{R})$ and $H_{b}^{n}(G ; \mathbb{R})$, respectively; for example, one could take the cohomological fundamental class of oriented closed connected hyperbolic $n$-manifolds and their fundamental groups, respectively [12]. Hence, the bounded cohomology functors $H_{b}^{n}(\cdot ; \mathbb{R})$ are not representable on the

- (homotopy) category of topological spaces

- (homotopy) category of finite CW-complexes

- category of groups

- category of finitely generated groups

- category of finitely presented groups

$-\ldots$

Notice that in view of the mapping theorem for bounded cohomology [12, 14], the pictures for the case of path-connected spaces and groups are equivalent. 


\subsection{Weak flexibility and countable additivity.}

Corollary 4.5 (finite functorial semi-norms and countable additivity). Let $C$ be a category such that for every object $X \in \mathrm{Ob}(C)$ the countably infinite coproduct $\coprod_{\mathbb{N}} X$ exists in $C$. Let $F: C \longrightarrow A b$ be a contravariant functor such that for all $X \in \mathrm{Ob}(C)$ the structure maps $X \longrightarrow \bigsqcup_{\mathbb{N}} X$ of the summands induce an isomorphism

$$
\varphi_{X}: F\left(\coprod_{\mathbb{N}} X\right) \rightarrow \prod_{\mathbb{N}} F(X) .
$$

If $\widehat{F}: C \longrightarrow \operatorname{snAb} b^{\text {fin }}$ is a finite homogeneous functorial semi-norm on $C$, then $\widehat{F}$ is trivial.

Proof. Let $\alpha \in F(X)$. It suffices to show that $\alpha$ is weakly flexible with respect to $F$ (Proposition 3.4). Let $Y:=\bigsqcup_{\mathbb{N}} X$, and let

$$
\beta:=\varphi_{X}^{-1}\left((d \cdot \alpha)_{d \in \mathbb{N}}\right) \in F(Y) .
$$

For $d \in \mathbb{N}$ the structure map $f_{d} \in \operatorname{Mor}_{C}\left(X, \bigsqcup_{\mathbb{N}} X\right)$ of the $d$-th summand satisfies

$$
F\left(f_{d}\right)(\beta)=F\left(f_{d}\right)\left(\varphi_{X}^{-1}\left((d \cdot \alpha)_{d \in \mathbb{N}}\right)\right)=F\left(\operatorname{id}_{X}\right)(d \cdot \alpha)=d \cdot \alpha .
$$

Hence, $\alpha$ is weakly flexible with respect to $F$.

Example 4.6 (additive cohomology theories). Corollary 4.5 applies to all generalised cohomology theory functors Top $\longrightarrow$ Ab that satisfy the countable additivity axiom; this includes, in particular, for all $n \in \mathbb{N}$ and all Abelian groups $A$ singular cohomology $H^{n}(\cdot ; A)$ : Top $\longrightarrow$ Ab in degree $n$.

Example 4.7 (group cohomology). Group cohomology does not admit a nontrivial finite homogeneous functorial semi-norm in non-zero degree: In the category of groups, all coproducts exist (given by free products of groups), and for $n \in \mathbb{N}_{>0}$ and all Abelian groups $A$ the group cohomology functor $H^{n}(\cdot ; A)$ : Group $\longrightarrow$ Ab satisfies the corresponding compatibility condition of Corollary 4.5, Notice that $H^{n}(\cdot ; A):$ Group $\longrightarrow A b$ in general is not representable if $n>1$.

Example 4.8 (comparison maps). Let $n \in \mathbb{N}_{>1}$. Because Abelian groups are amenable, we have that $H_{b}^{n}\left(K\left(\mathbb{Z}^{n}, 1\right) ; \mathbb{R}\right) \cong 0$ (which follows by averaging over invariant means [12, 14]), but $H^{n}\left(K\left(\mathbb{Z}^{n}, 1\right) ; \mathbb{R}\right) \cong \mathbb{R}$. This simple example shows that the comparison map $H_{b}^{n}(\cdot ; \mathbb{R}) \Longrightarrow H^{n}(\cdot ; \mathbb{R})$ in general is not surjective on the category of topological spaces or groups, respectively.

In contrast, we will now provide an argument that relies on the nontriviality of the $\ell^{\infty}$-semi-norm: Let $C$ be a category of spaces or groups having the following properties:

(1) The functor $H^{n}(\cdot ; \mathbb{R}): C \longrightarrow$ Ab does not admit a non-trivial finite homogeneous functorial semi-norm, and

(2) there exist $X \in \mathrm{Ob}(C)$ and $\varphi \in H^{n}(X ; \mathbb{R})$ with $0<\|\varphi\|_{\infty}<\infty$.

E.g., we could take the category of topological spaces or the category of groups. Moreover, let $c: H_{b}^{n}(\cdot ; \mathbb{R}) \Longrightarrow H^{n}(\cdot ; \mathbb{R})$ be the comparison map 
on $C$ (Example 2.11). Then, for all $X \in \mathrm{Ob}(C)$ we obtain a homogeneous semi-norm

$$
\begin{aligned}
H^{n}(X ; \mathbb{R}) & \longrightarrow \mathbb{R}_{\geq 0} \cup\{\infty\} \\
\varphi & \longmapsto \inf \left\{\|\psi\|_{\infty} \mid \psi \in H_{b}^{n}(X ; \mathbb{R}), c_{X}(\psi)=\varphi\right\}
\end{aligned}
$$

on $H^{n}(X ; \mathbb{R})$, which is finite if and only if $c_{X}: H_{b}^{n}(X ; \mathbb{R}) \longrightarrow H^{n}(X ; \mathbb{R})$ is surjective (recall that inf $\varnothing=\infty$ ). By construction of bounded cohomology, this semi-norm coincides with $\|\cdot\|_{\infty}$ on $H^{n}(X ; \mathbb{R})$. Hence, property (2) implies that this functorial semi-norm on $H^{n}(\cdot ; \mathbb{R}): C \longrightarrow A b$ is nontrivial. Therefore, property (1) implies that there is an $X \in \mathrm{Ob}(C)$, for which the comparison map $c_{X}: H_{b}^{n}(X ; \mathbb{R}) \longrightarrow H^{n}(X ; \mathbb{R})$ is not surjective.

It would be interesting to know whether such arguments could also be successfully applied to continuous bounded cohomology of restrictive classes of topological groups.

Example 4.9 (KK-theory of separable $C^{*}$-algebras). Let $B$ be a separable $C^{*}$ algebra. Then $K K(\cdot, B): C^{*} \longrightarrow$ Ab satisfies the countable additivity condition of Corollary 4.5] [7, Theorem 19.7.1], where $C^{*}$ denotes the category of separable $C^{*}$-algebras. Hence, $K K(\cdot, B): C^{*} \longrightarrow$ Ab does not admit a non-trivial finite homogeneous functorial semi-norm.

\section{WEAK FLEXIBILITY IN SINGULAR HOMOLOGY AND $\ell^{1}$-HOMOLOGY}

We will give characterisations of weak flexibility in singular homology (Section 5.1) and $\ell^{1}$-homology (Section 5.2) in terms of $\ell^{1}$-semi-norms. Moreover, we will show that flexibility in $\ell^{1}$-homology is equivalent to vanishing of classes (Section 5.3).

5.1. Weak flexibility in singular homology. Weak flexibility in singular homology can be characterised as follows:

Theorem 5.1 (weak flexibility in singular homology). Let $X$ be a topological space, let $n \in \mathbb{N}$, and let $\alpha \in H_{n}(X ; \mathbb{Z})$. Then the following are equivalent:

(1) The class $\alpha$ is weakly flexible with respect to $H_{n}(\cdot ; \mathbb{Z})$.

(2) The sequence $\left(\|d \cdot \alpha\|_{1, \mathbb{Z}}\right)_{d \in \mathbb{N}}$ contains a bounded subsequence.

For the proof of the theorem we will use that singular homology classes can be represented essentially in terms of combinatorial types that describe how faces of singular simplices in a singular chain can cancel in the singular chain complex:

Definition 5.2 (combinatorial types and associated chains).

- Let $k, n \in \mathbb{N}$. We then write

$$
T(k, n):=P\left((\{1, \ldots, k\} \times\{0, \ldots, n\})^{\times 2}\right)
$$

for the set of all relations on $\{1, \ldots, k\} \times\{0, \ldots, n\}$.

- For $t \in T(k, n)$, we define the topological space

$$
Y_{t}:=\{1, \ldots, k\} \times \Delta^{n} / \sim_{t},
$$


where " $\sim_{t}$ " is the equivalence relation generated by

$$
\begin{aligned}
(j, x) \sim_{t}\left(j^{\prime}, x^{\prime}\right) \Longleftrightarrow \exists_{y \in \Delta^{n-1}} \exists_{\ell, \ell^{\prime} \in\{0, \ldots, n\}} & i_{\ell}(y)=x \\
\wedge & i_{\ell^{\prime}}(y)=x^{\prime} \\
& \wedge\left((j, \ell),\left(j^{\prime}, \ell^{\prime}\right)\right) \in t .
\end{aligned}
$$

Here, $i_{\ell}: \Delta^{n-1} \longrightarrow \Delta^{n}$ denotes the inclusion of the $\ell$-th face.

- For $t \in T(k, n)$ and $\varepsilon \in\{-1,0,1\}^{k}$ we consider the singular chain

$$
z_{t, \varepsilon}:=\sum_{j=1}^{k} \varepsilon_{j} \cdot \tau_{j} \in C_{n}\left(Y_{t} ; \mathbb{Z}\right),
$$

where $\tau_{j}: \Delta^{n} \longrightarrow Y_{t}$ is the singular simplex induced from the inclusion $\Delta^{n} \hookrightarrow\{1, \ldots, k\} \times \Delta^{n}$ as $j$-th component.

Lemma 5.3 (representing singular classes through combinatorial types). Let $X$ be a topological space, and let $c=\sum_{j=1}^{k} \varepsilon_{j} \cdot \sigma_{j} \in C_{n}(X ; \mathbb{Z})$ be a cycle with $\varepsilon_{1}, \ldots, \varepsilon_{k} \in\{-1,0,1\}$. Let

$$
\begin{gathered}
t:=\left\{\left((j, \ell),\left(j^{\prime}, \ell^{\prime}\right)\right) \mid j, j^{\prime} \in\{1, \ldots, k\}, \ell, \ell^{\prime} \in\{0, \ldots, n\},\right. \\
\left.\sigma_{j} \circ i_{\ell}=\sigma_{j^{\prime}} \circ i_{\ell^{\prime}}\right\} \in T(k, n),
\end{gathered}
$$

and let $f_{c}: Y_{t} \longrightarrow X$ be the continuous map induced from the singular simplices $\sigma_{1}, \ldots, \sigma_{k}: \Delta^{n} \longrightarrow X$. Then $f_{c}$ is well-defined, $z_{t, \varepsilon} \in C_{n}\left(Y_{t} ; \mathbb{Z}\right)$ is a cycle, and

$$
H_{n}\left(f_{c} ; \mathbb{Z}\right)\left[z_{t, \varepsilon}\right]=[c]
$$

holds in $H_{n}(X ; \mathbb{Z})$.

Proof. All these properties directly follow from the construction.

The proof of Theorem 5.1 now is only a matter of counting:

Proof (of Theorem [5.1). Suppose that the class $\alpha$ is weakly flexible with respect to $H_{n}(\cdot ; \mathbb{Z})$, i.e., there is a space $Y$ and a class $\beta \in H_{n}(Y ; \mathbb{Z})$ such that $D(\beta, \alpha)$ is infinite. Let $d \in D(\beta, \alpha)$; then there is a map $f: Y \longrightarrow X$ with $H_{n}(f ; \mathbb{Z})(\beta)=d \cdot \alpha$; in particular,

$$
\||d| \cdot \alpha\|_{1, \mathbb{Z}}=\left\|H_{n}(f ; \mathbb{Z})(\beta)\right\|_{1, \mathbb{Z}} \leq\|\beta\|_{1, \mathbb{Z}} .
$$

Because $D(\beta, \alpha)$ is infinite, $\left(\|d \cdot \alpha\|_{1, \mathbb{Z}}\right)_{d \in \mathbb{N}}$ thus contains a subsequence that is bounded by $\|\beta\|_{1, \mathbb{Z}}$.

Conversely, suppose that the sequence $\left(\|d \cdot \alpha\|_{1, \mathbb{Z}}\right)_{d \in \mathbb{N}}$ contains a subsequence, say $\left(\left\|d_{m} \cdot \alpha\right\|_{1, \mathbb{Z}}\right)_{m \in \mathbb{N}^{\prime}}$ bounded by $k \in \mathbb{N}$. For $m \in \mathbb{N}$ let $c_{m} \in C_{n}(X ; \mathbb{Z})$ be a cycle representing $d_{m} \cdot \alpha$ with $\left\|c_{m}\right\|_{1, \mathbb{Z}} \leq k$; we may assume that $c_{m}$ is of the form

$$
c_{m}=\sum_{j=1}^{k} \varepsilon_{m, j} \cdot \sigma_{m, j}
$$

with $\varepsilon_{m, 1}, \ldots, \varepsilon_{m, k} \in\{-1,0,1\}$. Moreover, let $t_{m} \in T(k, n)$ be the corresponding combinatorial type of $c_{m}$ as in Lemma 5.3. Because the set

$$
T(k, n) \times\{-1,0,1\}^{k}
$$


is finite, there is a subsequence of $\left(c_{m}\right)_{m \in \mathbb{N}}$ of chains that have the same combinatorial type $t \in T(k, n)$ and the same coefficients $\varepsilon \in\{-1,0,1\}^{k}$. For any index $m \in \mathbb{N}$ of this subsequence, we have

$$
H_{n}\left(f_{c_{m}} ; \mathbb{Z}\right)\left[z_{t, \varepsilon}\right]=H_{n}\left(f_{\mathcal{c}_{m}} ; \mathbb{Z}\right)\left[z_{t_{m}, \varepsilon_{m}}\right]=\left[c_{m}\right]=d_{m} \cdot \alpha,
$$

where $f_{c_{m}}: Y_{t_{m}}=Y_{t} \longrightarrow X$ is the map from Lemma 5.3, Thus, $D\left(\left[z_{t, \varepsilon}\right], \alpha\right)$ is infinite, i.e., $\alpha$ is weakly flexible with respect to $H_{n}(\cdot ; \mathbb{Z})$.

Remark 5.4. The second condition of Theorem 5.1 is related to the vanishing of the $\ell^{1}$-semi-norm on singular homology with $\mathbb{R}$-coefficients as follows: If $X$ is a topological space, then for all $n \in \mathbb{N}$ the change of coefficients map $H_{n}(X ; \mathbb{Q}) \longrightarrow H_{n}(X ; \mathbb{R})$ is isometric with respect to corresponding $\ell^{1}$-semi-norms [24, Lemma 2.9]. Rearranging denominators hence shows that

$$
\left\|\alpha_{\mathbb{R}}\right\|_{1}=\inf _{d \in \mathbb{N}_{>0}} \frac{1}{d} \cdot\|d \cdot \alpha\|_{1, \mathbb{Z}}
$$

for all $\alpha \in H_{n}(X ; \mathbb{Z})$, where $\alpha_{\mathbb{R}} \in H_{n}(X ; \mathbb{R})$ denotes the image of $\alpha$ under the change of coefficients map.

This is the first step in a programme that defines and studies secondary invariants associated with the $\ell^{1}$-semi-norm and simplicial volume.

Corollary 5.5 (weak flexibility in singular homology, manifold case). Let $M$ be an oriented closed connected n-manifold. Then the following are equivalent:

(1) There exists an oriented closed connected n-manifold $N$ such that the set $\{\operatorname{deg} f \mid f \in \operatorname{map}(N, M)\}$ of mapping degrees is infinite.

(2) The fundamental class $[M]$ is weakly flexible with respect to $H_{n}(\cdot ; \mathbb{Z})$.

(3) The $\mathbb{R}$-fundamental class $[M]_{\mathbb{R}}$ is weakly flexible with respect to $H_{n}(\cdot ; \mathbb{R})$.

(4) The sequence $\left(\|d \cdot[M]\|_{1, \mathbb{Z}}\right)_{d \in \mathbb{N}}$ contains a bounded subsequence.

(5) All finite homogeneous functorial semi-norms on $H_{n}(\cdot ; \mathbb{R})$ are zero on $[M]_{\mathbb{R}}$.

Proof. By definition of the mapping degree and the universal coefficient theorem, the first three conditions are equivalent. In view of Theorem 5.1, condition (2) and condition (4) are equivalent. Proposition 3.4 and explicit constructions [9, Section 7.1] of functorial semi-norms on $H_{n}(\cdot ; \mathbb{R})$ show that condition (1) and condition (5) are equivalent.

More specifically, the case of bound 1 in Corollary 5.5(4) is equivalent to domination by an odd-dimensional sphere [19, Theorem 3.2].

Remark 5.6 (weak flexibility in group homology). Gaifullin's construction of aspherical URC manifolds [10] and the fact that $\|\cdot\|_{1, \mathbb{Z}}$ on integral singular homology of classifying/aspherical spaces coincides with $\|\cdot\|_{1, \mathbb{Z}}$ on integral group homology of the corresponding fundamental group (Example 2.6) allow to translate Theorem 5.1 also into the corresponding characterisation for weak flexbility with respect to $H_{n}(\cdot ; \mathbb{Z}):$ Group $\longrightarrow$ Ab.

5.2. Weak flexibility in $\ell^{1}$-homology. We will now discuss weak flexibility in $\ell^{1}$-homology of simplicial sets. In particular, the following discussions will apply to $\ell^{1}$-homology of spaces and groups. 
Setup 5.7. Let $C$ be a category, let $S: C \longrightarrow \Delta$ (Set) be a functor, and $n \in \mathbb{N}_{>0}$. We abbreviate

$$
H_{n}^{\ell^{1}, S}:=H_{n} \circ \mathrm{Ch}_{\mathbb{R}}^{\ell^{1}} \circ S: C \longrightarrow \mathrm{Ab} .
$$

This functor is equipped with the finite functorial $\ell^{1}$-semi-norm (Example 2.4). For simplicity, we assume that $C$ contains an object $\bullet$ and that the simplicial set $S(\bullet)$ corresponds to the simplicial set $P$ satisfying $P(n)=\{\varnothing\}$ for all $n \in \mathbb{N}$ (i.e., the simplicial set corresponding to a one-point space).

Definition 5.8 (replicable, pointable). In the situation of Setup 5.7, an object $X \in \mathrm{Ob}(C)$ is called

- replicable if the coproduct $\bigsqcup_{\mathbb{N}} X$ exists in $C$,

- pointable (with respect to $S$ ) if $\operatorname{Mor}_{C}(\bullet, X)$ and $\operatorname{Mor}_{C}(X, \bullet)$ are nonempty.

All (non-empty) topological spaces are replicable and pointable (with respect to the total singular complex functor) in the category of topological spaces. All groups are replicable and pointable (with respect to the simplicial classifying space functor) in the category of groups.

In $\ell^{1}$-homology, weak flexibility is the universal reason for vanishing of the $\ell^{1}$-semi-norm:

Theorem 5.9 (weak flexibility in $\ell^{1}$-homology). In the situation of Setup 5.7 let $X \in \mathrm{Ob}(C)$ be an object that is replicable and pointable, and let $\alpha \in H_{n}^{\ell^{1}, S}(X)$. Then the following are equivalent:

(1) The class $\alpha$ is weakly flexible with respect to $H_{n}^{\ell^{1}, S}$.

(2) All finite homogeneous functorial semi-norms on $H_{n}^{\ell^{1}, S}$ are zero on $\alpha$.

(3) We have $\|\alpha\|_{1}=0$.

Proof. The first condition implies the second one by Proposition 3.4. Clearly, the second condition implies the third.

For the remaining implication, suppose that $\|\alpha\|_{1}=0$. We show that $\alpha$ is weakly flexible with respect to $H_{n}^{\ell^{1}, S}$ : Because $X$ is pointable there exist morphisms $i \in \operatorname{Mor}_{C}(\bullet, X)$ and $q \in \operatorname{Mor}_{C}(X, \bullet)$. Let

$$
p:=i \circ q \in \operatorname{Mor}_{C}(X, X) \text {. }
$$

This morphism allows us to neglect certain $\ell^{1}$-chains: Using that $n \in \mathbb{N}_{>0}$, that $p$ factors over $\bullet$ and spelling out $\mathrm{Ch}_{\mathbb{R}}^{\ell^{1}}(S(\bullet))=\mathrm{Ch}_{\mathbb{R}}^{\ell^{1}}(P)$ explicitly yields:

$(*)$ For any cycle $c \in \mathrm{Ch}_{\mathbb{R}}^{\ell^{1}}(S(X))$ there is a chain $b \in \mathrm{Ch}_{\mathbb{R}}^{\ell^{1}}(S(X))$ satisfying

$$
\partial_{n+1} b=\mathrm{Ch}_{\mathbb{R}}^{\ell^{1}}(S(p))(c) \text { and }\|b\|_{1} \leq\|c\|_{1} .
$$

We now construct a suitable $\ell^{1}$-homology class on $Y:=\bigsqcup_{\mathbb{N}} X$ : For $k \in$ $\mathbb{N}$ let $i_{k} \in \operatorname{Mor}_{C}\left(X, \bigsqcup_{\mathbb{N}} X\right)$ be the structure map of the $k$-th summand. Using the universal property of the coproduct, for every $m \in \mathbb{N}$ we obtain a morphism $p_{m} \in \operatorname{Mor}_{C}(Y, X)$ satisfying for all $k \in \mathbb{N}$ :

$$
p_{m} \circ i_{k}= \begin{cases}\operatorname{id}_{X} & \text { if } k=m \\ p & \text { if } k \neq m\end{cases}
$$


Because of $\|\alpha\|_{1}=0$, for every $k \in \mathbb{N}$ there is a cycle $c_{k} \in \mathrm{Ch}_{\mathbb{R}}^{\ell^{1}} \circ S(X)$ representing $\alpha$ with

$$
\left\|c_{k}\right\|_{1} \leq \frac{1}{2^{k}}
$$

Hence,

$$
c:=\sum_{k \in \mathbb{N}} k \cdot \mathrm{Ch}_{\mathbb{R}}^{\ell^{1}}\left(S\left(i_{k}\right)\right)\left(c_{k}\right)
$$

is a well-defined $\ell^{1}$-chain in $\mathrm{Ch}_{\mathbb{R}}^{\ell^{1}}(S(Y))$, which is a cycle. We consider the corresponding class $\beta:=[c] \in H_{n}^{\ell^{1}, S}$.

The morphisms $\left(p_{k}\right)_{k \in \mathbb{N}}$ witness that $D(\beta, \alpha)$ is infinite: Because of property $(*)$, for every $k \in \mathbb{N}$ there is a chain $b_{k} \in \mathrm{Ch}_{\mathbb{R}}^{\ell^{1}}(S(X))$ with

$$
\partial_{n+1} b_{k}=\mathrm{Ch}_{\mathbb{R}}^{\ell^{1}}(S(p))\left(c_{k}\right) \quad \text { and } \quad\left\|b_{k}\right\|_{1} \leq\left\|c_{k}\right\|_{1} \leq \frac{1}{2^{k}} .
$$

Therefore, for all $m \in \mathbb{N}$ we have in $H_{n}^{\ell^{1}, S}(X)$ :

$$
\begin{aligned}
H_{n}^{\ell^{1}, S}\left(p_{m}\right)(\beta) & =\left[\sum_{k \in \mathbb{N}} k \cdot \mathrm{Ch}_{\mathbb{R}}^{\ell^{1}}\left(S\left(p_{m} \circ i_{k}\right)\right)\left(c_{k}\right)\right] \\
& =\left[m \cdot c_{m}\right]+\left[\sum_{k \in \mathbb{N} \backslash\{m\}} k \cdot \partial_{n+1} b_{k}\right] \\
& =m \cdot\left[c_{m}\right]+0 \\
& =m \cdot \alpha .
\end{aligned}
$$

In the third step, we used that $\sum_{k \in \mathbb{N} \backslash\{m\}} k \cdot \partial_{n+1} b_{k}$ is indeed an $\ell^{1}$-chain and that $\sum_{k \in \mathbb{N} \backslash\{m\}} k \cdot \partial_{n+1} b_{k}=\partial_{n+1}\left(\sum_{k \in \mathbb{N} \backslash\{m\}} k \cdot b_{k}\right)$.

Hence, $\alpha$ is weakly flexible with respect to $H_{n}^{\ell^{1}, S}$.

Moreover, using the same type of arguments, we also obtain that the $\ell^{1}$-semi-norm is the "maximal" functorial semi-norm on $\ell^{1}$-homology:

Proposition 5.10 (finite functorial semi-norms on $\ell^{1}$-homology). In the situation of Setup 5.7. suppose that all countable coproducts exist in $C$ and that all objects in $C$ are pointable. Then any finite homogeneous functorial semi-norm on $H_{n}^{\ell^{1}, S}$ is dominated by a multiple of the $\ell^{1}$-semi-norm.

Proof. Let $|\cdot|$ be a finite homogeneous functorial semi-norm on $H_{n}^{\ell^{1}, S}$. Assume for a contradiction that $|\cdot|$ is not dominated by a multiple of the $\ell^{1}$ semi-norm. That is, for every $k \in \mathbb{N}$ there exists an object $X_{k} \in \mathrm{Ob}(C)$ and a class $\alpha_{k} \in H_{n}^{\ell^{1}, S}\left(X_{k}\right)$ satisfying

$$
\left|\alpha_{k}\right|>k \cdot 2^{k} \cdot\left\|\alpha_{k}\right\|_{1} .
$$

By Theorem 5.9, $\left\|\alpha_{k}\right\|_{1} \neq 0$; moreover, because $|\cdot|$ and $\|\cdot\|_{1}$ are homogeneous, we can renormalise these classes in such a way that we may assume in addition that

$$
\frac{1}{2} \leq\left\|\alpha_{k}\right\|_{1} \leq 1
$$

for all $k \in \mathbb{N}$. In particular, for every $k \in \mathbb{N}$ there is a cycle $c_{k} \in \mathrm{Ch}_{\mathbb{R}}^{\ell^{1}}\left(S\left(X_{k}\right)\right)$ representing $\alpha_{k}$ with $\left\|c_{k}\right\|_{1} \leq 2$. 
Let $X:=\bigsqcup_{k \in \mathbb{N}} X_{k}$ and let $i_{k} \in \operatorname{Mor}_{C}\left(X_{k}, X\right)$ be the structure map of the $k$-th summand. Furthermore, let $q_{k} \in \operatorname{Mor}_{C}\left(X_{k}, \bullet\right)$ and $j_{k} \in \operatorname{Mor}_{C}\left(\bullet, X_{k}\right)$. Using the universal property of the coproduct, for every $m \in \mathbb{N}$ we obtain a morphism $p_{m} \in \operatorname{Mor}_{C}\left(X, X_{m}\right)$ satisfying for all $k \in \mathbb{N}$ :

$$
p_{m} \circ i_{k}= \begin{cases}\operatorname{id}_{X_{m}} & \text { if } k=m \\ j_{m} \circ q_{k} & \text { if } k \neq m .\end{cases}
$$

Then

$$
\beta:=\left[\sum_{k \in \mathbb{N}} \frac{1}{2^{k}} \cdot \mathrm{Ch}_{\mathbb{R}}^{\ell^{1}}\left(S\left(i_{k}\right)\right)\left(c_{k}\right)\right] \in H_{n}^{\ell^{1}, S}(X)
$$

is a well-defined $\ell^{1}$-homology class and the same argument as in the proof of Theorem 5.9 shows that

$$
H_{n}^{\ell^{1}, S}\left(p_{m}\right)(\beta)=\frac{1}{2^{m}} \cdot \alpha_{m}
$$

for all $m \in \mathbb{N}$. Therefore, for all $m \in \mathbb{N}$ we obtain

$$
|\beta| \geq\left|H_{n}^{\ell^{1}, S}\left(p_{m}\right)(\beta)\right|=\frac{1}{2^{m}} \cdot\left|\alpha_{m}\right|>m \cdot\left\|\alpha_{m}\right\|_{1} \geq \frac{1}{2} \cdot m
$$

which contradicts $|\cdot|$ being finite.

The corresponding statement for singular homology is not true: it is possible to "distort" the $\ell^{1}$-semi-norm in such a way that the resulting functorial semi-norm is finite, but not dominated by a multiple of the $\ell^{1}$-seminorm [9, proof of Theorem 5.7].

Moreover, for the sake of completeness, we also mention the following, qualitative, version of Lemma 5.3 for $\ell^{1}$-homology of spaces:

Lemma 5.11 (countable geometric support of $\ell^{1}$-homology classes). Let $X$ be a topological space, let $n \in \mathbb{N}$, and let $\alpha \in H_{n}^{\ell^{1}}(X ; \mathbb{R})$. Then there exists a countable simplicial complex $K$, a class $\beta \in H_{n}^{\ell^{1}}(|K| ; \mathbb{R})$ and a continuous map $f:|K| \longrightarrow X$ with

$$
H_{n}^{\ell^{1}}(f ; \mathbb{R})(\beta)=\alpha \text { and }\|\beta\|_{1}=\|\alpha\|_{1} .
$$

Proof. We give a direct, geometric proof: Let $\left(c_{k}\right)_{k \in \mathbb{N}} \in C_{n}^{\ell^{1}}(X ; \mathbb{R})$ be a sequence of cycles representing $\alpha$ in $H_{n}^{\ell^{1}}(X ; \mathbb{R})$ with

$$
\|\alpha\|_{1}=\inf _{k \in \mathbb{N}}\left\|c_{k}\right\|_{1},
$$

and let $\left(b_{k}\right)_{k \in \mathbb{N}} \in C_{n+1}^{\ell^{1}}(X ; \mathbb{R})$ satisfying

$$
\partial_{n+1} b_{k}=c_{0}-c_{k}
$$

for these chains $\left(c_{k}\right)_{k \in \mathbb{N}}$ and $\left(b_{k}\right)_{k \in \mathbb{N}}$, in total only a countable set of singular simplices of dimension $k$ or $k+1$ is needed. Similarly to Lemma 5.3 and the discussion preceding that lemma, we can construct a topological space $Y$ by gluing a countable set of copies of $\Delta^{n}$ and $\Delta^{n+1}$ according to the combinatorics of the relations between the respective faces given by the fact that the $\left(c_{k}\right)_{k \in \mathbb{N}}$ are cycles and by Equation (1); moreover, using the simplices in $Y$ and the same coefficients as in the original chains on $X$, we obtain corresponding chains $\left(\bar{c}_{k}\right)_{k \in \mathbb{N}} \subset C_{k}^{\ell^{1}}(Y ; \mathbb{R})$, and $\left(\bar{b}_{k}\right)_{k \in \mathbb{N}} \subset C_{k+1}^{\ell^{1}}(Y ; \mathbb{R})$, 
as well as a continuous map $f: Y \longrightarrow X$ (constructed out of the singular simplices of the original chains) satisfying

$$
\begin{aligned}
\left\|\bar{c}_{k}\right\|_{1} & =\left\|c_{k}\right\|_{1}, \\
\partial_{n} \bar{c}_{k} & =0, \\
\partial_{n+1} \bar{b}_{k} & =\bar{c}_{0}-\bar{c}_{k}, \\
C_{n}^{\ell^{1}}(f)\left(\bar{c}_{0}\right) & =c_{0} .
\end{aligned}
$$

Hence, $\beta \in H_{n}(Y ; \mathbb{R})$ satisfies $H_{n}^{\ell^{1}}(f ; \mathbb{R})(\beta)=\alpha$. Functoriality of the $\ell^{1}$-semi-norm and the above relations between the constructed cycles on $Y$ show that $\|\beta\|_{1}=\|\alpha\|_{1}$.

The double barycentric subdivision of the simplices involved in the construction of $Y$ yields a countable simplicial complex $K$ with $|K| \cong Y$.

Already in degree 0 one can see that not every $\ell^{1}$-homology class of any topological space can be isometrically represented by an $\ell^{1}$-homology class of a finite simplicial complex.

5.3. Flexibility in $\ell^{1}$-homology. On the other hand, in $\ell^{1}$-homology, flexibility of a class is the same as being trivial; clearly, this behaviour is very different from ordinary homology.

Theorem 5.12 (flexibility in $\ell^{1}$-homology). In the situation of Setup 5.7 let $X \in \mathrm{Ob}(C)$, and let $\alpha \in H_{n}^{\ell^{1}, S}(X)$. Then the following are equivalent:

(1) The class $\alpha$ is flexible with respect to $H_{n}^{\ell^{1}}$, .

(2) The class $\alpha$ is dominated by a class that is flexible with respect to $H_{n}^{\ell^{1}, S}$, i.e., there is an object $Y \in \mathrm{Ob}(C)$, a flexible class $\beta \in H_{n}^{\ell^{1}, S}(Y)$, and a morphism $f \in \operatorname{Mor}_{C}(Y, X)$ with $H_{n}^{\ell^{1}, S}(f)(\beta)=\alpha$.

(3) We have $\alpha=0 \in H_{n}^{\ell^{1}, S}(X)$.

Proof. Clearly, the third condition implies the first, and the first condition implies the second.

Suppose that the second condition holds, i.e., there is $Y \in \mathrm{Ob}(C)$, a flexible class $\beta \in H_{n}^{\ell^{1}, S}(Y)$, and $f \in \operatorname{Mor}_{C}(Y, X)$ with $H_{n}^{\ell^{1}, S}(f)(\beta)=\alpha$. By functoriality, it suffices to show that $\beta=0 \in H_{n}^{\ell^{1}, S}(Y)$.

Because $\beta$ is flexible with respect to $H_{n}^{\ell^{1}, S}$ there is a $d \in \mathbb{Z}$ with $|d| \geq 2$ and an endomorphism $g \in \operatorname{Mor}_{C}(Y, Y)$ satisfying

$$
H_{n}^{\ell^{1}, S}(g)(\beta)=d \cdot \beta \text {. }
$$

Let $c \in \mathrm{Ch}_{\mathbb{R}}^{\ell^{1}}(S(Y))$ be a cycle representing the class $\beta$. Hence, there is a chain $b \in \mathrm{Ch}_{\mathbb{R}}^{\ell^{1}}(S(Y))$ with

$$
\partial_{n+1}(b)=c-\frac{1}{d} \cdot \mathrm{Ch}_{\mathbb{R}}^{\ell^{1}}(S(g))(c) .
$$

Then the "geometric series"

$$
b^{\prime}:=\sum_{k \in \mathbb{N}} \frac{1}{d^{k}} \cdot \mathrm{Ch}_{\mathbb{R}}^{\ell^{1}}\left(S\left(g^{\circ k}\right)\right)(b)
$$


is an $\ell^{1}$-chain. Rearranging absolutely convergent series together with functoriality of $\mathrm{Ch}_{\mathbb{R}}^{\ell^{1}} \circ S$ shows that

$$
\partial_{n+1} b^{\prime}=\sum_{k \in \mathbb{N}} \frac{1}{d^{k}} \cdot \mathrm{Ch}_{\mathbb{R}}^{\ell^{1}}\left(S\left(g^{\circ k}\right)\right)(c)-\sum_{k \in \mathbb{N}} \frac{1}{d^{k+1}} \cdot \mathrm{Ch}_{\mathbb{R}}^{\ell^{1}}\left(S\left(g^{\circ k+1}\right)\right)(c)=c
$$

holds in $\mathrm{Ch}_{\mathbb{R}}^{\ell^{1}}(S(Y))$. Therefore, $\beta=[c]=\left[\partial_{n+1} b^{\prime}\right]=0$ in $H_{n}^{\ell^{1}, S}(Y)$.

So, in $\ell^{1}$-homology, the difference between trivial $\ell^{1}$-semi-norm and vanishing of the class corresponds to the difference between weak flexibility and flexibility. It is an open problem to determine whether for any topological space $X$, any $n \in \mathbb{N}$ and any $\alpha \in H_{n}(X ; \mathbb{R})$ with $\|\alpha\|_{1}=0$ the image of $\alpha$ in $H_{n}^{\ell^{1}}(X ; \mathbb{R})$ under the comparison map between singular and $\ell^{1}$-homology can be non-trivial. This problem plays a role in the context of simplicial volume of non-compact manifolds [18, Theorem 6.4].

\section{REFERENCES}

[1] J.F. Adams. A variant of E. H. Brown's representability theorem, Topology, 10, pp. 185198, 1971. Cited on page:8

[2] M. Amann. Mapping degrees of self-maps of simply-connected manifolds, preprint (to appear in International Mathematics Research Notices), arXiv:1109.0960 [math.AT], 2011. Cited on page: 7

[3] M. Arkowitz, G. Lupton. Rational obstruction theory and rational homotopy sets, Math. Z., 235(3), pp. 525-539, 2000. Cited on page: 7

[4] U. Bader, A. Furman, R. Sauer. Efficient subdivision in hyperbolic groups and applications, Groups, Geometry, and Dynamics, 7, pp. 263-292, 2013. Cited on page:

[5] R. Benedetti, C. Petronio. Lectures on Hyperbolic Geometry, Universitext, Springer, 1992. Cited on page: 40

[6] T. Bühler. On the algebraic foundations of bounded cohomology, Mem. Amer. Math. Soc., 214 (1006), 2011. Cited on page:56

[7] B. Blackadar. K-Theory for Operator Algebras, MSRI Publications, 5, Cambridge University Press, 1998. Cited on page:10

[8] C. Costoya, A. Viruel. Every finite group is the group of self homotopy equivalences of an elliptic space, Acta Mathematica, 213, pp. 49-62, 2014. Cited on page: 7

[9] D. Crowley, C. Löh. Functorial semi-norms on singular homology and (in)flexible manifolds, preprint (to appear in Algebraic $\mathcal{E}$ Geometric Topology), arXiv:1103.4139 [math.GT], 2011. Cited on page:1,4, 6, 12,15

[10] A. Gaifullin. Universal realisators for homology classes, Geom. Topol., 17(3), pp. 17451772, 2013. Cited on page:12

[11] P.G. Goerss, J.F. Jardine. Simplicial Homotopy Theory, Birkhäuser, 2010. Cited on page:3, 4

[12] M. Gromov. Volume and bounded cohomology, Publ. Math. IHES, 56, pp. 5-99, 1982. Cited on page:1, 6, 7,8

[13] M. Gromov. Metric Structures for Riemannian and Non-Riemannian Spaces with appendices by M. Katz, P. Pansu, and S. Semmes, translated by S. M. Bates. Progress in Mathematics, 152, Birkhäuser, 1999. Cited on page:1

[14] N.V. Ivanov. Foundations of the theory of bounded cohomology, J. Soviet Math., 37, pp. 1090-1114, 1987. Cited on page: 6, 8, 9

[15] D. Kotschick, C. Löh. Fundamental classes not representable by products, J. London Math. Soc., 79(3), pp. 545-561, 2009. Cited on page:1 1

[16] J.-F. Lafont, B. Schmidt. Simplicial volume of closed locally symmetric spaces of noncompact type, Acta Math., 197, p. 129-143, 2006. Cited on page:14 4

[17] C. Löh. Simplicial Volume, Bull. Man. Atl., pp. 7-18, 2011 Cited on page:4

[18] C. Löh. Isomorphisms in $\ell^{1}$-homology, Münster J. of Math., 1, pp. 237-266, 2008. Cited on page: 17 
[19] C. Löh. Odd manifolds of small integral simplicial volume, preprint, 2015. Cited on page: 12

[20] C. Löh, C. Pagliantini. Integral foliated simplicial volume of hyperbolic 3-manifolds, preprint (to appear in Groups, Geometry, and Dynamics), arXiv:1403.4518 [math.GT], 2014. Cited on page:5

[21] C. Löh, R. Sauer. Degree theorems and Lipschitz simplicial volume for non-positively curved manifolds of finite volume, Journal of Topology, 2, pp. 193-225, 2009. Cited on page:11 4

[22] N. Monod. Continuous Bounded Cohomology of Locally Compact Groups, Lecture Notes in Mathematics, 1758, Springer, 2001. Cited on page: 6

[23] H. J. Munkholm. Simplices of maximal volume in hyperbolic space, Gromov's norm, and Gromov's proof of Mostow's rigidity theorem (following Thurston). In Topology Symposium, Siegen 1979, Lecture Notes in Mathematics, 788, pp. 109-124, Springer, 1980.

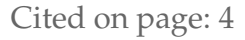

[24] M. Schmidt. $L^{2}$-Betti Numbers of $\mathcal{R}$-spaces and the Integral Foliated Simplicial Volume, $\mathrm{PhD}$ thesis, WWU Münster, 2005.

Available online at http://nbn-resolving.de/urn:nbn:de:hbz:6-05699458563 Cited on page:12

[25] J. Strom. Modern Classical Homotopy Theory, Graduate Studies in Mathematics, 127, AMS, 2011. Cited on page:8

[26] W. P. Thurston. The geometry and topology of 3-manifolds, mimeographed notes, 1979. Available online at http://www.msri.org/publications/books/gt3m. Cited on page: 4 [

\section{Clara Löh}

Fakultät für Mathematik

Universität Regensburg

93040 Regensburg

Germany

clara.loeh@mathematik.uni-regensburg.de

http://www.mathematik.uni-regensburg.de/loeh 